\title{
Relación entre la actividad física durante el recreo escolar, actividad física semanal
} y expediente académico

\section{Relationship between physical activity during school recess, weekly physical activity and academic performance}

\section{Martí Xavier Bennàsser Torrandell ${ }^{1}$; Josep Vidal Conti ${ }^{2}$}

1 Consejería de Educación, Universidad e Investigación. Illes Balears, España.

2 Grupo de Investigación en Ciencias de la Actividad Física y el Deporte. Universitat de les Illes Balears, España

Contacto: josep.vidal@uib.es

Cronograma editorial: Artículo recibido: 23/07/2020 Aceptado: 14/12/2020 Publicado: 01/01/2021 https://doi.org/10.17979/sportis.2021.7.1.6850 


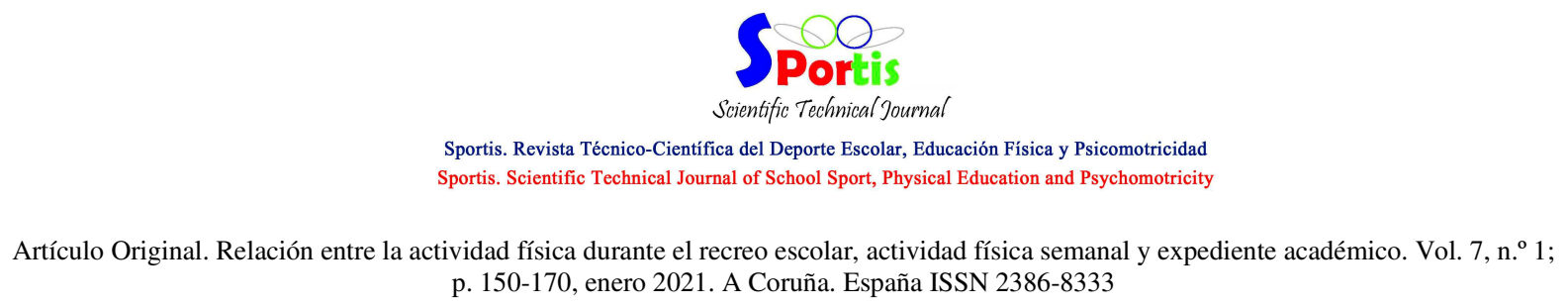
p. 150-170, enero 2021. A Coruña. España ISSN 2386-8333

\title{
Resumen
}

La inactividad física implica importantes consecuencias para la salud. Los recreos escolares representan una oportunidad para la práctica de actividad física. El objetivo de este estudio fue analizar la relación entre la actividad física realizada durante el recreo escolar, la actividad física semanal y el expediente académico de los jóvenes. En el estudio participaron 2.399 jóvenes de entre 10 y 16 años, de los cuales 1.175 fueron chicos (49\%) y 1.224 chicas (51\%), con una edad media de 12,7 años. En colaboración con diferentes centros educativos se les administró un cuestionario de hábitos de práctica deportiva en jóvenes, que incluía las siguientes variables: percepción de ser personas activas durante los recreos, índice de masa corporal y horas de actividad física y de pantalla semanales. Con el fin de estimar el volumen máximo de oxígeno, se administró el test de course-navette, y además el centro educativo aportó el expediente académico. El estudio muestra que la percepción de los jóvenes de ser activos durante los recreos se relaciona con menores IMC $(t=66,458 ; p=<0,001)$ y VO2 máx. ( $t=27,165 ; p=<0,001)$ más saludables, menos horas de pantalla $(t=51,659 ; p=<$ $0,001)$, más horas de actividad física semanal $(t=16,684 ; p=<0,001)$ y mejores expedientes académicos $(t=42,944 ; p=<0,001)$. Estos resultados refuerzan la necesidad de establecer estrategias de promoción y de gestión de entornos, que favorezcan el aumento de la actividad física durante los recreos.

\section{Palabras clave}

Actividad física; salud; recreo; escuela; rendimiento académico.

\begin{abstract}
:
Physical inactivity has significant health consequences. School recess represent an opportunity to practice physical activity. The objective of this study was to analyze the relationship between the physical activity during school recess, weekly physical activity, and academic performance in young people. The study included 2399 young people aged 10 to 16 years, (1.175 boys, $49 \%$ - and 1.224 girls, 51\%) with a mean age of 12,7 years. In collaboration with different educational centers, a sports habits practice questionnaire in young people was administered among them, that included the following variables: perception of being active people during recess, body mass index, performed physical activity hours, and weekly screen hours. The course-navette test, was administered in order to estimate the maximum volume of oxygen, besides the academic dossier of students was provided by the school. The study shows that young people's perception of being active people during recess, correlates with healthier BMI $(t=66,458 ; p=<0,001)$ and VO2 max. $(t$ $=27,165 ; p=<0,001)$, with less screen hours $(t=51,659 ; p=<0,001)$, more weekly physical activity $(t=16,684 ; p=<0,001)$, and better academic performance $(t=42,944 ; p=<0,001)$.

Para citar este artículo utilice la siguiente referencia: Bennàsser Torrandell, M.X. y Vidal Conti, J. (2021). Relación entre la actividad física durante el recreo escolar, actividad física semanal y expediente académico. Sportis Sci J, 7 (1), 150-170.

https://doi.org/10.17979/sportis.2021.7.1.6850

http://revistas.udc.es/
\end{abstract}


Artículo Original. Relación entre la actividad física durante el recreo escolar, actividad física semanal y expediente académico. Vol. 7, n. ${ }^{\circ} 1$; p. 150-170, enero 2021. A Coruña. España ISSN 2386-8333

These results reinforce the need for establish promoting and management environments strategies that boost the increase of physical activity during recess.

\section{Keywords}

Physical activity; health; recess; school; academic performance. 


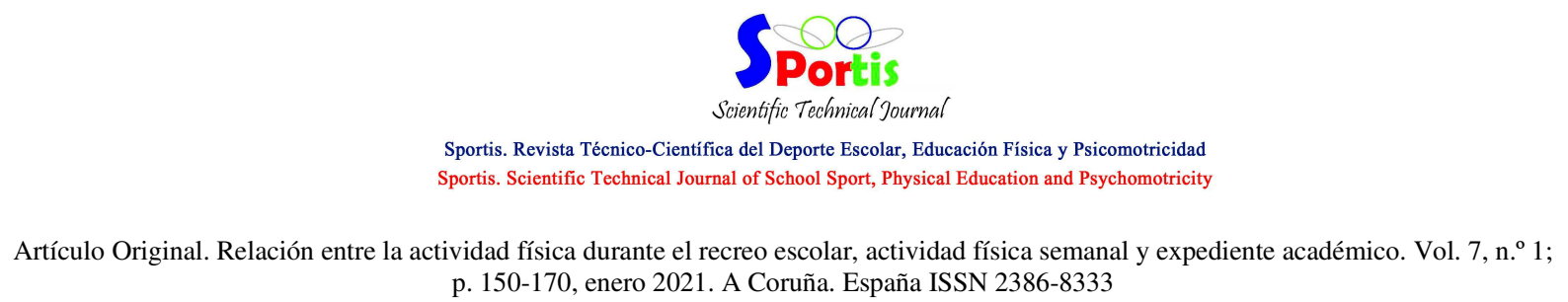

\section{Introducción}

La falta de actividad física ha sido calificada por la Organización Mundial de la Salud (OMS, 2017) como un problema de salud pública, con importantes consecuencias para la salud. A nivel mundial el 84,7\% de las chicas de entre 11 y 17 años, no cumplen la recomendación mínima de 60 minutos de actividad física diaria establecida por la OMS (OMS, 2010), y el porcentaje de chicos es del 77,6\% (Guthold, Stevens, Riley \& Bull, 2020). A nivel español el estudio ANIBES, indica que el 73\% de las chicas de entre 9 y 17 años, no cumplen esa recomendación, mientras que el porcentaje de chicos es del 44,5 \% (Mielgo et al., 2016).

Asociado a la inactividad física se encuentra el sobrepeso y la obesidad. Un problema de magnitud creciente en muchos países, influyendo considerablemente en la prevalencia de enfermedades no transmisibles y en la salud general, representando el cuarto factor de riesgo de mortandad más importante en todo el mundo (OMS, 2010). Otro factor destacable asociado a la inactividad física es el aumento del tiempo que pasan los jóvenes delante las pantallas (Lizandra, Devís, Valencia, Tomás \& Peiró, 2019). La OMS fija la recomendación en un máximo de 2 horas de pantalla diaria y según la encuesta nacional de salud 2017, en España menos del $50 \%$ de los jóvenes cumple con esa recomendación, y menos del $25 \%$ en fin de semana (Ministerio de Sanidad, Consumo y Bienestar Social, 2019). El estudio EPOIB II, desarrollado por el Govern de les Illes Balears, indica que los jóvenes de las Illes Balears manifiestan pasar más de 2 horas diarias delante las pantallas, (Govern de les Illes Balears, 2018).

Para reducir esta tendencia seria clave la promoción de la actividad física entre la población y especialmente entre los jóvenes, ya que la actividad física juega un papel significativo en la prevención del sobrepeso y la obesidad (De Bourdeaudhuij et al., 2013). En este sentido la escuela puede ser un contexto adecuado y conveniente para la promoción de la actividad física y la salud, ya que los escolares pasan gran parte del día en él. (Aznar \& Webster, 2006).

Concretamente el recreo puede suponer entre el 5 y el $40 \%$ de los niveles diarios recomendados de actividad física, llegando hasta el $40 \%$ en los niños y hasta el $30 \%$ en las niñas (Ridgers, Stratton \& Fairclough, 2006). En los últimos años se ha prestado atención a la

Para citar este artículo utilice la siguiente referencia: Bennàsser Torrandell, M.X. y Vidal Conti, J. (2021). Relación entre la actividad física durante el recreo escolar, actividad física semanal y expediente académico. Sportis Sci J, 7 (1), 150-170.

https://doi.org/10.17979/sportis.2021.7.1.6850

http://revistas.udc.es/

153 


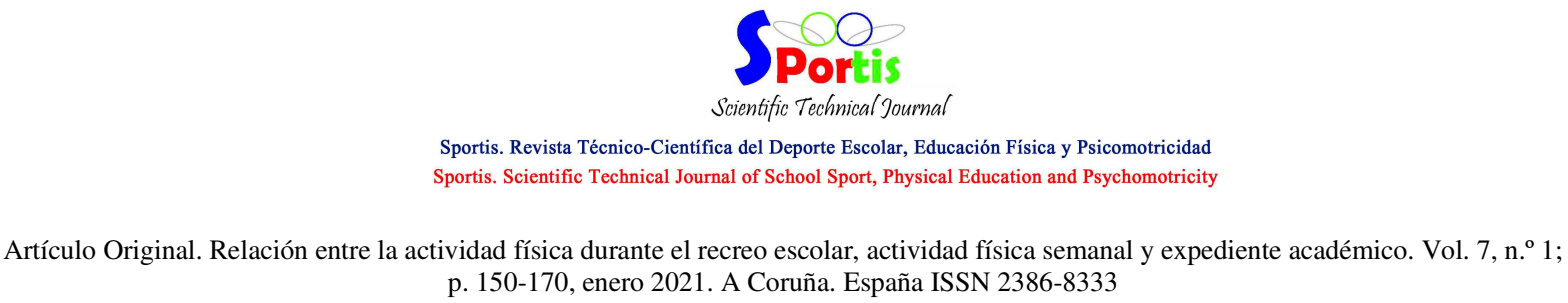
p. 150-170, enero 2021. A Coruña. España ISSN 2386-8333

implicación de los recreos, en el desarrollo infantil en general y en la actividad física en particular (Shape, 2017). Los estudios se han centrado en conocer la cantidad de actividad física que realizan los jóvenes durante los recreos (Hall, Ochoa, Macías, Zúñiga \& Sáenz, 2018), las interacciones sociales que se producen (Arias \& Martínez, 2018), o en examinar la efectividad (Parrish, Chong, Moriarty, Betterham \& Ridgers, 2020) o en facilitar intervenciones escolares para aumentar actividad física de los jóvenes durante el recreo (Salas \& Vidal, 2020). Además, otros estudios se han centrado en los elementos que facilitan o dificultan que los jóvenes sean físicamente activos durante los recreos (Stanley, Boshoff \& Dollman, 2012), incluyendo la perspectiva del género (Martínez, Bartolomé, Rodríguez, Pardo \& Martínez, 2017) y el sobrepeso (López, Molina, Chinchilla, Garrido \& Pascual, 2017). Estudios previos como los de Esteban et al., (2017) y Norris, Van Steen, Direito \& Stamatakis, (2019), se han centrado en conocer las relaciones entre la actividad física que realizan los jóvenes en el recreo, y su respectivo expediente académico.

Hasta el momento no tenemos conocimiento de ningún estudio transversal en jóvenes, que relacione la actividad física realizada durante los recreos, con el VO2 máx. y el tiempo de pantalla y que relacione a la vez otras variables como el IMC, las horas de actividad física, y el expediente académico.

Conocer estas relaciones será de gran interés para establecer los vínculos entre la actividad física desarrollada en los recreos, y diferentes variables relacionadas con la salud y el rendimiento académico, favoreciendo así la necesidad de desarrollar estrategias de promoción de la actividad física en los recreos.

Así pues, el presente estudio se plantea la siguiente hipótesis: los chicos y las chicas que perciben ser más activos durante los recreos tienen un menor IMC, un mejor expediente académico, un VO2 máx. más alto, pasan menos tiempo delante de las pantallas, y realizan más horas de actividad física durante la semana.

Para citar este artículo utilice la siguiente referencia: Bennàsser Torrandell, M.X. y Vidal Conti, J. (2021). Relación entre la actividad física durante el recreo escolar, actividad física semanal y expediente académico. Sportis Sci J, 7 (1), 150-170.

https://doi.org/10.17979/sportis.2021.7.1.6850 http://revistas.udc.es/ 


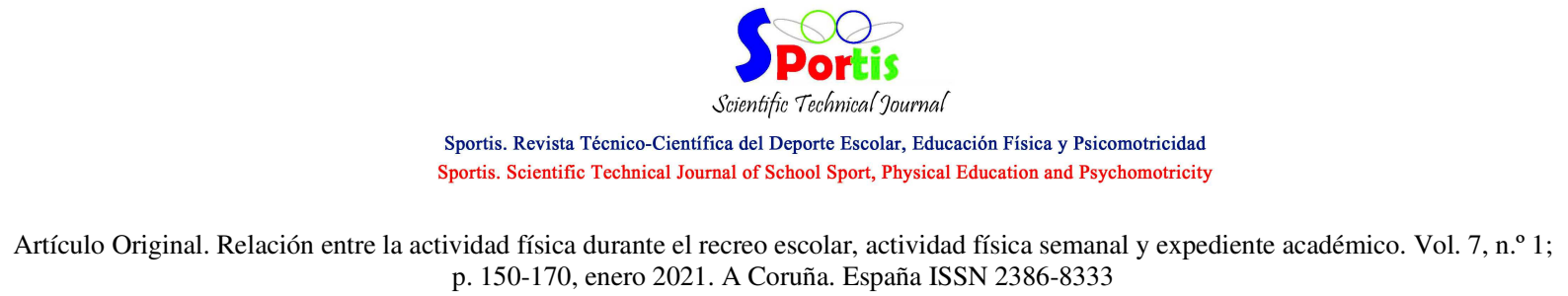
p. 150-170, enero 2021. A Coruña. España ISSN 2386-8333

\section{Material y Métodos}

\section{Participantes}

Se realizó un estudio de carácter transversal mediante encuesta, con una muestra representativa de la población de Mallorca de entre 10 y 16 años de edad. La población objeto de estudio fueron escolares de educación secundaria obligatoria (ESO) y tercer ciclo de educación primaria de los colegios de Mallorca. La estimación del tamaño muestral para una población infinita determinó una muestra teórica de 1.066 participantes, con un nivel de confianza del $95 \%$ y un error muestral predeterminado del 3\%. Se envió una carta a todos los centros educativos de Mallorca con la invitación a su participación, y de entre aquellos que aceptaron, se informó por carta a los padres / madres de los participantes, de su carácter voluntario, sobre la naturaleza y el propósito del estudio y se requirió el consentimiento informado por escrito.

La muestra se obtuvo por conglomerados (centros escolares) y muestreo intencional durante el curso 2014/2015. El estudio se llevó a cabo con una muestra final de 2.399 participantes, (error muestral del 1,6\% y nivel de confianza del 95\%), de los cuales 1.175 fueron chicos (49\%) y 1.224 chicas $(51 \%)$, con una edad media de 12,7 años (DT 1,9). En relación con la distribución por edades de las personas 256 tenían 10 años (10,7\%), 557 tenían 11 años (23,2\%), 416 tenían 12 años (17,3\%), 318 tenían 13 años (13,3\%), 318 tenían 14 años (13,3\%), 302 tenían 15 años (12,6\%), y 232 tenían 16 años (9,6\%) (Tabla 1). 
Tabla 1: descriptivos

\begin{tabular}{|c|c|c|c|}
\hline Variable & Pregunta / descripción & Categorización de respuestas & $\mathbf{N} \%$ \\
\hline \multirow[t]{2}{*}{ Género } & \multirow[t]{2}{*}{ Auto reportado por el alumno/a. } & Chicas & $1.175(49,0)$ \\
\hline & & Chicos & $1.224(51,0)$ \\
\hline \multirow[t]{7}{*}{ Edad } & \multirow[t]{7}{*}{ Auto reportado por el alumno/a. } & 10 años & $256(10,7)$ \\
\hline & & 11 años & $557(23,2)$ \\
\hline & & 12 años & $416(17,3)$ \\
\hline & & 13 años & $318(13,3)$ \\
\hline & & 14 años & $318(13,3)$ \\
\hline & & 15 años & $302(12,6)$ \\
\hline & & 16 años & $232(9,6)$ \\
\hline \multirow[t]{4}{*}{ IMC } & \multirow{4}{*}{$\begin{array}{l}\text { Altura y peso auto reportados } \\
\text { por el alumnado. }\end{array}$} & Infrapeso & $238(11,6)$ \\
\hline & & Normopeso & $1.268(61,5)$ \\
\hline & & Sobrepeso & $434(21,0)$ \\
\hline & & Obesidad & $122(5,9)$ \\
\hline \multirow{6}{*}{$\begin{array}{l}\text { Actividad Física } \\
\text { durante el recreo }\end{array}$} & \multirow{6}{*}{$\begin{array}{l}\text { ¿Qué haces normalmente } \\
\text { durante el tiempo de recreo en } \\
\text { la escuela? }\end{array}$} & Personas activas & $966(47,0)$ \\
\hline & & Chicas & $364(37,7)$ \\
\hline & & \multirow[b]{2}{*}{ Personas sedentarias } & $602(62,3)$ \\
\hline & & & $1089(53,0)$ \\
\hline & & Chicas & $672(61,7)$ \\
\hline & & Chicos & $417(38,3)$ \\
\hline
\end{tabular}

\section{Instrumentos}

Para la recogida de información se administraron los cuestionarios "School Health Action, Planning and Evaluation System (SHAPES)" (Wong, Leatherdale \& Manske, 2006) a niños y niñas (alfa de Cronbach $=0,633$ ) para las variables de tiempo de pantalla, actividad física, peso y altura, y el cuestionario de hábitos alimentarios de la población escolar para los/as niños/as (Programa PERSEO: estrategia NAOS) para la variable de actividad física durante el tiempo de patio. El expediente académico se obtuvo del programa de gestión académica de los centros escolares. Además, se utilizó la prueba de campo denominada test de Léger, también conocida como test de Course Navette para obtener el VO2 máx. (Léger \& Lambert, 1982), ampliamente documentada su validez y fiabilidad (Jódar, 2003).

Las variables analizadas en el presente estudio fueron: sexo (masculino / femenino); edad (año de nacimiento); altura $(\mathrm{cm})$; peso (kg); IMC; actividad física (horas semanales); VO2 
Artículo Original. Relación entre la actividad física durante el recreo escolar, actividad física semanal y expediente académico. Vol. 7, n. ${ }^{\circ}$ 1; p. 150-170, enero 2021. A Coruña. España ISSN 2386-8333
\end{abstract}

máx. (ml/Kg/min); percepción de ser una persona activa o sedentaria en los recreos, tiempo de pantalla (horas semanales) y expediente académico (Tabla 1 y Tabla 2).

\title{
Procedimiento
}

Todos los participantes fueron previamente informados de los objetivos del estudio y su protocolo. Los cuestionarios se administraron por parte de encuestadores formados específicamente para este estudio y con la colaboración de los docentes de los centros educativos. Los cuestionarios fueron distribuidos entre los participantes, adjuntándose las pertinentes instrucciones de uso para su correcta utilización. El IMC fue autoreportado por los propios participantes. Durante las clases de educación física se llevó a cabo el test de CourseNavette, y el centro educativo aportó los resultados del expediente académico.

\section{Análisis estadístico}

Para los análisis descriptivos se calcularon medias y desviaciones típicas para las variables continuas y para las categóricas se estimaron frecuencias. La normalidad de las variables fue determinada con la prueba de Shapiro-Wilk, dado que las condiciones se cumplieron se llevó a cabo pruebas paramétricas.

Para la comparación de variables continuas se utilizó la prueba t-Student y correlaciones bivariadas. Para estudiar la relación entre las diferentes variables se realizaron análisis de regresión logística, con el correspondiente cálculo de odds ratio (OR) y el 95\% de intervalo de confianza (IC). El nivel de significación establecido fue $\mathrm{p}<0,05$.

Todos los análisis fueron realizados mediante el paquete estadístico SPSS-23 para Windows.

\section{Resultados}

Los resultados obtenidos a partir de los cuestionarios y pruebas aplicadas muestran que 966 (47\%) de los jóvenes apreciaron ser activos durante los recreos y 1089 (53\%) ser sedentarios. En la tabla 1 además, se refleja las medidas de IMC obtenidas por los jóvenes, determinando;

Para citar este artículo utilice la siguiente referencia: Bennàsser Torrandell, M.X. y Vidal Conti, J. (2021). Relación entre la actividad física durante el recreo escolar, actividad física semanal y expediente académico. Sportis Sci J, 7 (1), 150-170.

https://doi.org/10.17979/sportis.2021.7.1.6850 http://revistas.udc.es/ 
Artículo Original. Relación entre la actividad física durante el recreo escolar, actividad física semanal y expediente académico. Vol. 7, n. ${ }^{\circ}$ 1; p. 150-170, enero 2021. A Coruña. España ISSN 2386-8333

238 participantes con infrapeso (11,6\%), 1268 con normopeso (61,5\%), 434 con sobrepeso (21\%) y 122 con obesidad $(5,9 \%)$.

Los jóvenes que perciben ser activos durante los recreos, realizan 11.54 horas a la semana de media respecto a las 9,12 horas de aquellos que no lo perciben $(t=16,684 ; p=\langle 0,001)$. Igualmente, estos mismos jóvenes, obtienen un menor IMC ( $t=66,458 ; p=<0,001)$, un mejor expediente académico $(t=42,944 ; p=<0,001)$, un mayor nivel de VO2 máx. $(t=$ $27,165 ; p=<0,001)$, pasan menos tiempo delante de las pantallas $(t=51,659 ; p=<0,001) \mathrm{y}$ realizan más horas de actividad física semanal $(t=16,684 ; p=<0,001)$. (tabla 2).

Tabla 2. Diferencias entre personas activas y sedentarias en los recreos.

\begin{tabular}{|c|c|c|c|c|c|c|}
\hline & \multicolumn{2}{|c|}{ Total } & \multicolumn{2}{|c|}{ Chicos } & \multicolumn{2}{|c|}{ Chicas } \\
\hline & Sedentarias & Activas & Sedentarias & Activas & Sedentarias & Activas \\
\hline IMC & $\begin{array}{l}20,19 \\
(3,57)\end{array}$ & $\begin{array}{c}18,88 \\
(3,18) *\end{array}$ & $\begin{array}{l}20,44 \\
(3,29)\end{array}$ & $\begin{array}{c}19,09 \\
(3,27) *\end{array}$ & $\begin{array}{l}20,03 \\
(3,73)\end{array}$ & $\begin{array}{c}15,51 \\
(2,96) *\end{array}$ \\
\hline VO2 máx. & $\begin{array}{c}36,88 \\
(10,11)\end{array}$ & $\begin{array}{c}41,18 \\
(11,70) *\end{array}$ & $\begin{array}{l}36,62 \\
(6,18)\end{array}$ & $\begin{array}{l}37,05 \\
(5,33)\end{array}$ & $\begin{array}{l}30,80 \\
(4,97)\end{array}$ & $\begin{array}{c}34,71 \\
(4,39) *\end{array}$ \\
\hline $\begin{array}{l}\text { Tiempo de } \\
\text { Pantalla } \\
\text { (h/sem). }\end{array}$ & $\begin{array}{c}24,36 \\
(16,35)\end{array}$ & $\begin{array}{c}18,00 \\
(12,00) *\end{array}$ & $\begin{array}{c}25,05 \\
(17,16)\end{array}$ & $\begin{array}{c}18,75 \\
(13,33) *\end{array}$ & $\begin{array}{c}23,91 \\
(15,81)\end{array}$ & $\begin{array}{c}16,50 \\
(11,88) *\end{array}$ \\
\hline $\begin{array}{l}\text { Actividad } \\
\text { Física (h/sem). }\end{array}$ & $\begin{array}{c}9,12 \\
(6,26)\end{array}$ & $\begin{array}{c}11,54 \\
(7,37) *\end{array}$ & $\begin{array}{l}10,32 \\
(6,42)\end{array}$ & $\begin{array}{c}12,89 \\
(7,66) *\end{array}$ & $\begin{array}{c}8,37 \\
(6,04)\end{array}$ & $\begin{array}{c}9,12 \\
(6,12)\end{array}$ \\
\hline $\begin{array}{l}\text { Expediente } \\
\text { académico }\end{array}$ & $\begin{array}{c}6,47 \\
(1,42)\end{array}$ & $\begin{array}{c}6,88 \\
(1,338) *\end{array}$ & $\begin{array}{c}6,16 \\
(1,44)\end{array}$ & $\begin{array}{c}6,75 \\
(1,40) *\end{array}$ & $\begin{array}{c}6,65 \\
(1,37)\end{array}$ & $\begin{array}{c}7,08 \\
(1,19) *\end{array}$ \\
\hline
\end{tabular}

Los datos muestran la media y la desviación típica

* La correlación es significativa al nivel 0,01

Respecto a la perspectiva de género, observamos que $602(62,3 \%)$ chicos y $364(37,7 \%)$ chicas apreciaron ser activas durante los recreos, frente a 417 (38,3\%) chicos y $672(61,7 \%)$ chicas que apreciaron ser sedentarias (tabla 1). En la tabla 2, se reflejan las medidas de IMC obtenidas por los jóvenes, determinando; que tanto las chicas $(t=38,443 ; p=<0,001)$ como 


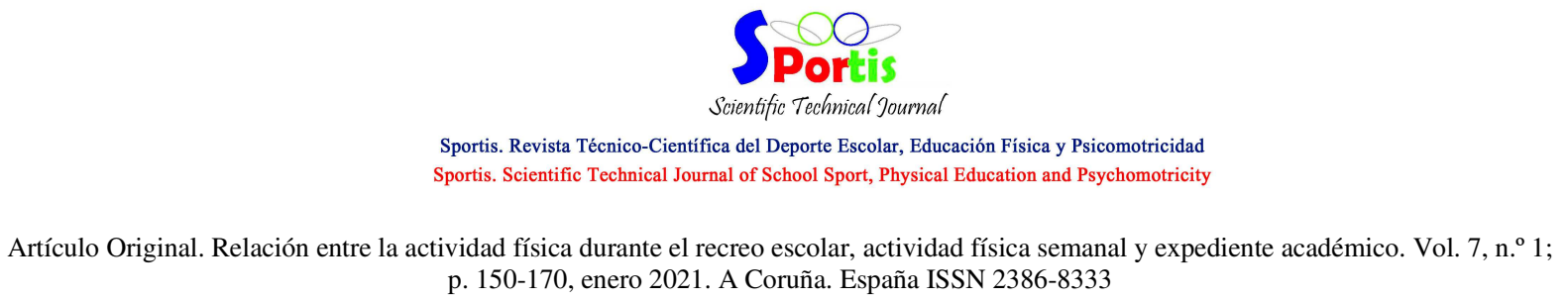
p. 150-170, enero 2021. A Coruña. España ISSN 2386-8333

los chicos $(t=36,425 ; p=<0,001)$, que perciben ser activos durante los recreos escolares, tienen menores IMC que aquellas chicas y chicos que no lo perciben.

La percepción de ser una persona activa durante los recreos escolares se relaciona con que los chicos realicen más horas semanales de actividad física $(t=23,358 ; p=<0,001)$, no así las chicas $(t=2,443 ; p=0,119)$ (tabla 2). En sentido inverso las chicas activas durante los recreos obtienen niveles de VO2 máx. ( $t=65,415 ; p=<0,001)$, significativamente superiores a las chicas que no perciben ser activas, no así los de los chicos $(t=547 ; p=0,460)$. (Tabla 2$)$.

En el resto de las variables, expediente académico y horas de pantalla, no se muestran diferencias entre género. (Tabla 2). Las chicas que perciben ser activas durante los recreos obtienen mejores expedientes académicos, que aquellas que no lo perciben $(t=23,244 ; p=<$ $0,001)$ igualmente los chicos $(t=42,232 ; p=<0,001)$. Del mismo modo las chicas pasan menos tiempo delante de las pantallas $(t=29,682 ; p=<0,001)$, igualmente que los chicos $(t=$ $25,537 ; p=<0,001)$.

La tabla 3 muestra la asociación entre la actividad física durante el recreo escolar y el IMC, la actividad física, el tiempo de pantalla, el VO2 máx. y el expediente académico, mediante el ajuste de un modelo de regresión logística binaria.

Sin diferenciar por género, tener un VO2max elevado, una cantidad elevada de actividad física y una cantidad baja de tiempo de pantalla se asocian con una mayor probabilidad de ser una persona activa durante el tiempo de recreo.

En el caso concreto de los chicos, dicha probabilidad aumenta cuando el IMC es bajo (OR = $0,98)$, la actividad física global es alta $(\mathrm{OR}=1,08)$ y el expediente académico es alto $(\mathrm{OR}=$ $1,13)$.

En el caso de las chicas, la probabilidad de ser activas durante el tiempo de patio aumenta cuando el VO2 máx. es alto $(\mathrm{OR}=1,19)$ y el tiempo de pantalla es bajo $(\mathrm{OR}=0,96)$.

Para citar este artículo utilice la siguiente referencia: Bennàsser Torrandell, M.X. y Vidal Conti, J. (2021). Relación entre la actividad física durante el recreo escolar, actividad física semanal y expediente académico. Sportis Sci J, 7 (1), 150-170.

https://doi.org/10.17979/sportis.2021.7.1.6850 http://revistas.udc.es/ 
Artículo Original. Relación entre la actividad física durante el recreo escolar, actividad física semanal y expediente académico. Vol. 7, n. ${ }^{\circ}$ 1; p. 150-170, enero 2021. A Coruña. España ISSN 2386-8333

Tabla 3. Asociación entre la actividad física durante el recreo escolar, IMC, actividad física, tiempo de pantalla, VO2 máx. y expediente académico.

\begin{tabular}{lcccccc}
\cline { 2 - 6 } & \multicolumn{2}{c}{ Total } & \multicolumn{2}{c}{ Chicos } & \multicolumn{2}{c}{ Chicas } \\
\cline { 2 - 7 } & OR (I.C. 95\%) & Sig. & OR (I.C. 95\%) & Sig. & OR (I.C. 95\%) & Sig. \\
\hline IMC & $0,98(0,92-1,05)$ & 0,598 & $0,89(0,81-0,98)$ & 0,021 & $1,08(0,97-1,20)$ & 0,147 \\
\hline VO2 máx. & $1,08(1,04-1,13)$ & $<0,001$ & $0,99(0,94-1,04)$ & 0,727 & $1,19(1,08-1,31)$ & 0,001 \\
\hline $\begin{array}{l}\text { Tiempo de } \\
\text { Pantalla (h/sem). }\end{array}$ & $0,98(0,96-0,99)$ & 0,005 & $0,99(0,97-1,01)$ & 0,338 & $0,96(0,93-0,99)$ & 0,021 \\
\hline $\begin{array}{l}\text { Actividad } \\
\text { Física (h/sem). }\end{array}$ & $1,08(1,04-1,12)$ & $<0,001$ & $1,10(1,04-1,16)$ & $<0,001$ & $1,03(0,95-1,12)$ & 0,466 \\
\hline $\begin{array}{l}\text { Expediente } \\
\text { académico }\end{array}$ & $1,13(0,95-1,34)$ & 0,165 & $1,24(1,00-1,55)$ & 0,050 & $1,03(0,74-1,43)$ & 0,871 \\
\hline
\end{tabular}

En la tabla 4 se muestran las correlaciones entre las distintas variables tanto con la muestra total como diferenciando por género. Pese a que los valores de correlación son bajos, destacar la correlación negativa entre los valores de IMC y VO2 máx. y positiva del expediente académico y VO2 máx. 
Artículo Original. Relación entre la actividad física durante el recreo escolar, actividad física semanal y expediente académico. Vol. 7, n. ${ }^{\circ}$ 1; p. 150-170, enero 2021. A Coruña. España ISSN 2386-8333

Tabla 4. Correlaciones totales y por género.

\begin{tabular}{lccccc}
\cline { 2 - 5 } Total & IMC & VO2 máx. & $\begin{array}{c}\text { T. Pantalla } \\
(\mathrm{h} / \mathrm{sem}) .\end{array}$ & AF (h/sem). & $\begin{array}{c}\text { Exp. } \\
\text { Académico }\end{array}$ \\
\hline IMC & 1 & $-0,31^{* *}$ & $0,18^{* *}$ & $-0,01$ & $-0,18^{* *}$ \\
VO2 máx. & & 1 & $-0,12^{* *}$ & $0,29^{* *}$ & $0,24^{* *}$ \\
T. Pantalla (h/sem). & & & 1 & $0,08^{* *}$ & $-0,23^{* *}$ \\
AF (h/sem). & & & 1 & $-0,05$ \\
Exp. Académico & & & & & 1 \\
\hline
\end{tabular}

\begin{tabular}{lccccc}
\cline { 2 - 5 } Chicos & IMC & VO2 máx. & $\begin{array}{c}\text { T. Pantalla } \\
(\mathrm{h} / \mathrm{sem}) .\end{array}$ & AF (h/sem). & $\begin{array}{c}\text { Exp. } \\
\text { Académico }\end{array}$ \\
\hline IMC & 1 & $-0,27^{* *}$ & $0,21^{* *}$ & $-0,02$ & $-0,18^{* *}$ \\
VO2 máx. & 1 & $-0,01$ & $0,26^{* *}$ & $0,13^{* *}$ \\
T. Pantalla (h/sem). & & & 1 & 0,05 & $-0,21^{* *}$ \\
AF (h/sem). & & & 1 & $-0,09^{*}$ \\
Exp. Académico & & & & & 1 \\
\hline
\end{tabular}

\begin{tabular}{lccccc}
\cline { 2 - 5 } Chicas & IMC & VO2 máx. & $\begin{array}{c}\text { T. Pantalla } \\
(\mathrm{h} / \mathrm{sem}) .\end{array}$ & $\mathrm{AF}(\mathrm{h} / \mathrm{sem})$. & $\begin{array}{c}\text { Exp. } \\
\text { Académico }\end{array}$ \\
\hline IMC & 1 & $-0,42 * *$ & $0,15 * *$ & 0,02 & $-0,18^{* *}$ \\
VO2 máx. & 1 & $-0,27^{* *}$ & $0,13^{*}$ & $0,44^{* *}$ \\
T. Pantalla (h/sem). & & & 1 & $0,14^{* *}$ & $-0,26^{* *}$ \\
AF (h/sem). & & & 1 & $-0,06$ \\
Exp. Académico & & & & & 1 \\
\hline
\end{tabular}

* La correlación es significativa al nivel 0,05.

** La correlación es significativa al nivel 0,01 .

\section{Discusión}

El estudio se plantea la hipótesis que consiste en que los chicos y las chicas que perciben ser más activos durante los recreos tienen un menor IMC, un mejor expediente académico, un VO2 máx. más alto, pasan menos tiempo delante de las pantallas, y realizan más horas de Para citar este artículo utilice la siguiente referencia: Bennàsser Torrandell, M.X. y Vidal Conti, J. (2021). Relación entre la actividad física durante el recreo escolar, actividad física semanal y expediente académico. Sportis Sci J, 7 (1), 150-170. https://doi.org/10.17979/sportis.2021.7.1.6850 http://revistas.udc.es/ 


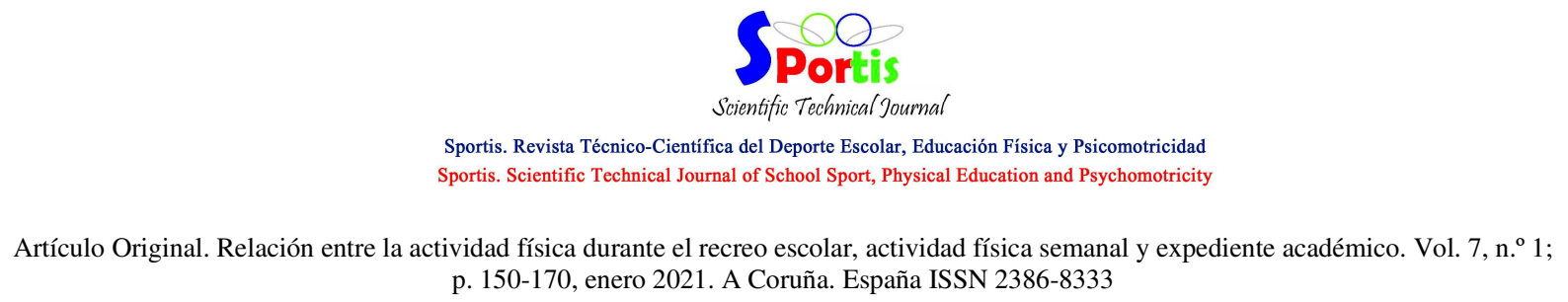
p. 150-170, enero 2021. A Coruña. España ISSN 2386-8333

actividad física durante la semana. Los resultados confirman esa hipótesis en la mayoría de sus variables.

La obtención de mejores IMC por parte de los jóvenes que perciben ser activos durante los recreos es un hecho relevante, teniendo en cuenta que unos niveles reducidos de IMC en la juventud implicaran niveles reducidos en la edad adulta (Ward et al., 2017). Esta asociación no coincide con el estudio de López et al. (2017), donde no se encontraron diferencias significativas entre la actividad física desarrollada en los recreos por niños de 6 a 8 años, con peso normal y con sobrepeso. En cambio, coincide parcialmente con los resultados del estudio de Brusseau et al. (2011), donde no se establecieron diferencias entre jóvenes con peso normal y sobrepeso en los recreos matinales de 15 minutos, pero en los recreos del almuerzo el alumnado con sobrepeso realizó menos actividad física que el alumnado con peso normal. Estos resultados sugieren que la percepción del nivel de actividad física en los recreos no es independiente del IMC.

En relación con el expediente académico, los resultados coinciden con el reciente estudio de Norris et al. (2019), que después de desarrollar una revisión sistemática y un meta análisis, concluyeron que las lecciones de actividad física crean un impacto positivo tanto en la actividad física como en los resultados educativos. Cabe destacar pero que en este estudio no se relacionan exclusivamente los resultados académicos con la actividad física en el recreo, sino que lo hace con las lecciones de actividad física en general. En cambio, los resultados no coinciden con el estudio de Esteban et al. (2017) que indica que el tiempo dedicado a diferentes intensidades de actividad física durante la educación física y el recreo, no afecta el rendimiento académico de los jóvenes. En este mismo estudio justifica esta falta de relación por la poca cantidad de actividad física que desarrollaron los jóvenes durante los recreos.

Se han realizado estudios que han analizado la práctica de actividad física de los jóvenes y su relación con el VO2 máx. (Martínez \& Sánchez, 2008; Ortega, Ruiz, Hurtig \& Sjöström, 2008), con el tiempo de pantalla (Abarca, Zaragoza, Generelo \& Julián, 2010; Serrano et al., 2011), y con los correlatos que influyen en su pràctica (Orlieb et al., 2013), pero hasta donde sabemos, ninguno había incluido la tipología de la actividad física en los recreos como Para citar este artículo utilice la siguiente referencia: Bennàsser Torrandell, M.X. y Vidal Conti, J. (2021). Relación entre la actividad física durante el recreo escolar, actividad física semanal y expediente académico. Sportis Sci J, 7 (1), 150-170. 


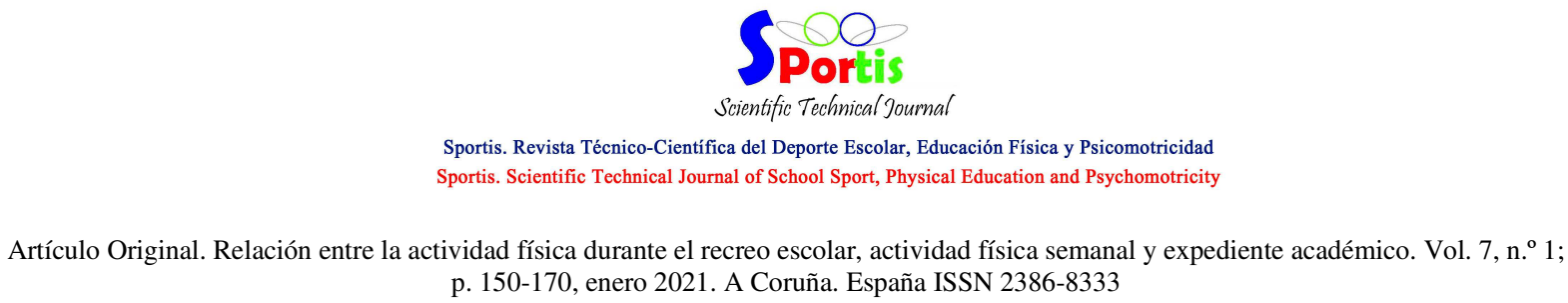
p. 150-170, enero 2021. A Coruña. España ISSN 2386-8333

posible variable asociada, lo que dificulta la comparación con el presente estudio. Es por este motivo que creemos que se abre una nueva ventana a explorar y que se requiere de un estudio de mayor profundidad al respecto.

El alumnado que percibe ser físicamente activo durante los recreos obtiene mayores valores de VO2 máx. Estos resultados coinciden con el estudio de Ortega et al. (2008), que afirma que los adolescentes físicamente activos presentan una mayor probabilidad de tener una capacidad cardiovascular saludable. En cambio, en el artículo de Martínez \& Sánchez (2008) se indica que, en la mayoría de los estudios, la relación entre actividad física y condición física es débil o moderada y en algunos, no es significativa. Asimismo, se destaca que esta débil asociación, podría producirse porque las medidas de actividad física y de condición física varían mucho en cuanto a su validez y fiabilidad.

Los resultados igualmente reflejan, que los jóvenes que son físicamente activos durante los recreos pasan menos horas delante las pantallas. El estudio de Serrano et al. (2011) nos indica que el tiempo total acumulado delante de las pantallas se asocia negativamente con el nivel de actividad física de los jóvenes y que la participación en actividades físicas organizadas parece contrarrestar el impacto negativo del tiempo excesivo frente a las pantallas en la actividad física. En este sentido la actividad física durante los recreos puede contribuir a contrarrestar ese impacto negativo. En cambio, el estudio de Abarca et al. (2010), pone de manifiesto la falta de relación entre la actividad física y el tiempo de pantalla.

La hipótesis también se cumple en relación a que los jóvenes que realizan más actividad física durante los recreos realizan más horas de actividad física durante la semana. Destacar que la actividad física durante los recreos representa un indicador de una mayor actividad física en general, convirtiendo estos espacios en un lugar fácil y accesible, tanto para la práctica de actividad física de los jóvenes, como para la identificación de los hábitos de actividad física fuera de ese entorno escolar. Por ese motivo el recreo debe facilitar un espacio de juego y un entorno con equipamiento adecuado para incentivar a que los jóvenes participen en mayor medida en prácticas de actividad física (Delidou, Matsouka \& Nikolaidis, 2016).

Para citar este artículo utilice la siguiente referencia: Bennàsser Torrandell, M.X. y Vidal Conti, J. (2021). Relación entre la actividad física durante el recreo escolar, actividad física semanal y expediente académico. Sportis Sci J, 7 (1), 150-170.

https://doi.org/10.17979/sportis.2021.7.1.6850

http://revistas.udc.es/ 


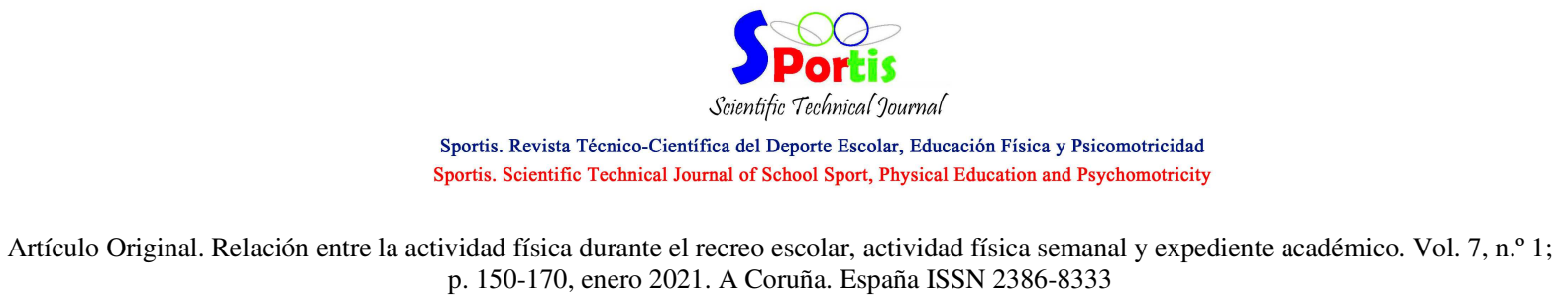
p. 150-170, enero 2021. A Coruña. España ISSN 2386-8333

Desde la perspectiva de género se observa que, en la misma línea de otros estudios, las chicas pasaron más tiempo en actividades sedentarias que los chicos durante el recreo (Martínez et al., 2017).

Cabe destacar que no hay diferencias entre chicos y chicas en 3 de las 5 variables estudiadas. Tanto las chicas como los chicos que perciben ser activos durante los recreos obtienen menores IMC, mejores expedientes académicos y pasan menos tiempo semanal delante las pantallas, que aquellos chicos y chicas que no lo perciben.

En cambio, sí se observan diferencias en las variables de actividad física diaria y VO2 máx. No se puede establecer una relación entre la percepción que tienen las chicas de ser activas durante los recreos y la actividad física semanal y en el caso de los chicos tampoco se puede establecer esa relación en el caso del VO2 máx.

El hecho de que los chicos no obtengan valores significativos en la relación entre la actividad física durante los recreos y el VO2 máx. y sí se establezca esa relación en el caso de las chicas, puede venir justificado porque la relación entre actividad física y condición física es débil o moderada y en algunos, no es significativa (Martínez \& Sánchez, 2008), y que hemos expuesto con anterioridad.

Tampoco se puede establecer una relación entre la percepción que tienen las chicas de ser activas durante los recreos y el hecho de realizar más horas de actividad física semanal. Este hecho podría venir provocado, porque los rasgos de cultura deportiva de las chicas en actividades deportivas no organizadas, es superior que en actividades organizadas, un 66\%, frente a un $55 \%$ en el caso de España y un $76 \%$ frente al $65 \%$ en el caso de las Illes Balears (Viñas \& Pérez, 2011). Estas actividades físicas no organizadas se podrían ver favorecidas en el contexto de los recreos, teniendo en cuenta además, que este tipo de práctica contribuye significativamente más en las chicas que en los chicos, al total de actividad física recomendada (Martínez, Aznar \& Contreras, 2015). En este sentido Lamoneda \& Huertas (2017) proponen el establecimiento de actuaciones relacionadas con la gestión de espacios y con el uso de metodologías cooperativas e inclusivas para facilitar su práctica.

Para citar este artículo utilice la siguiente referencia: Bennàsser Torrandell, M.X. y Vidal Conti, J. (2021). Relación entre la actividad física durante el recreo escolar, actividad física semanal y expediente académico. Sportis Sci J, 7 (1), 150-170.

https://doi.org/10.17979/sportis.2021.7.1.6850 http://revistas.udc.es/ 


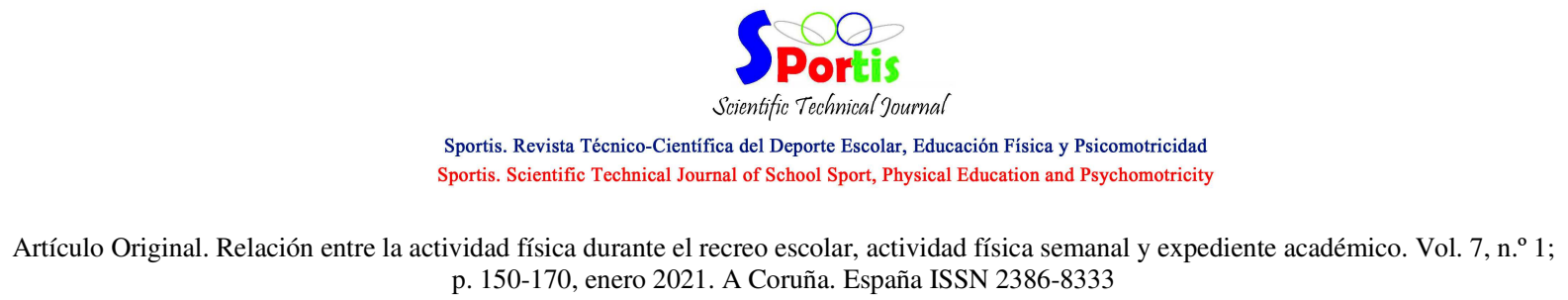
p. 150-170, enero 2021. A Coruña. España ISSN 2386-8333

Una de las mayores fortalezas del estudio, es la amplia muestra conseguida, cercana a los 2.399 participantes, que permite extraer unas conclusiones sólidas. La principal limitación en cambio es que la actividad física en el recreo fue auto-reportada por los jóvenes y no a través de métodos objetivos como los acelerómetros. La limitación de las medidas de auto-reporte reside en aspectos como sesgos de memoria, dificultades a la hora de interpretar las preguntas y modificaciones por un sesgo de deseabilidad social, aunque estudios como el de Argiropoulou, Michalopoulou, Aggloussis \& Avgerinos (2004), que compararon diferentes cuestionarios autoreportados para valorar la actividad física frente al acelerómetro, concluyeron la validez de estos instrumentos respecto al acelerómetro.

Otra limitación fue que el IMC se obtuvo a través del peso y talla auto declarados por los participantes, y aunque podría ser más adecuado haber sido pesados y tallados de forma objetiva, estudios como el de Sevane et al. (2013), muestran que su análisis como variable continua en jóvenes, conlleva un escaso margen de error.

En conclusión, el estudio pone de manifiesto que la percepción de los jóvenes de ser activos durante los recreos se relaciona con IMC y VO2 máx. más saludables, con menos horas de pantalla y con más horas actividad física semanal. Igualmente se puede concluir que un mayor nivel de VO2 máx., un menor tiempo de pantalla y la realización de más horas de actividad física semanal se asocia con la percepción de un mayor nivel de actividad física durante los recreos. Se evidencia pues, que la actividad física practicada por los jóvenes en los recreos escolares se vincula con mejores índices de salud y es por ello, que toda esta información refuerza la necesidad de establecer y reforzar políticas futuras sobre los períodos de recreo y su papel dentro del día a día escolar y hace recomendable el establecimiento de estrategias de intervención dirigidas al aumento de la actividad física de los jóvenes en este contexto.

\section{Referencias}

Abarca, A., Zaragoza, J., Generelo, E., \& Julián, J. A. (2010). Comportamientos sedentarios y patrones de actividad física en adolescentes. Revista Internacional de Medicina y Ciencias de

Para citar este artículo utilice la siguiente referencia: Bennàsser Torrandell, M.X. y Vidal Conti, J. (2021). Relación entre la actividad física durante el recreo escolar, actividad física semanal y expediente académico. Sportis Sci J, 7 (1), 150-170.

https://doi.org/10.17979/sportis.2021.7.1.6850

http://revistas.udc.es/ 


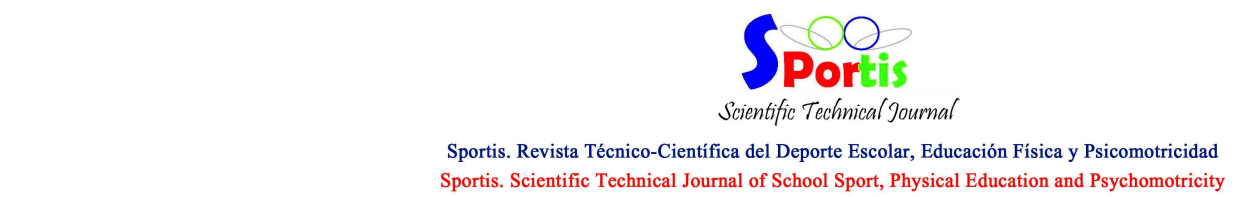

Artículo Original. Relación entre la actividad física durante el recreo escolar, actividad física semanal y expediente académico. Vol. 7, n. ${ }^{\circ}$ 1; p. 150-170, enero 2021. A Coruña. España ISSN 2386-8333

la Actividad Física y el Deporte, 10(39), 410427. http://cdeporte.rediris.es/revista/revista39/artcomportamientos170.htm

Argiropoulou, E. C., Michalopoulou, M., Aggloussis, N. \& Avgerinos, A. (2004). Validity and Reliability of Physical Activity Measures in Greek High School Age Children. Journal of Sports Science and Medicine. 3, 147-159.

Arias, T., \& Martínez, C. (2018). El juego dirigido en los recreos como método de inclusión del alumnado con necesidades especiales. Sportis. Scientific Journal of School Sport, Physical Education and Psychomotricity, 4(2), 388-408. https://doi.org/10.17979/sportis.2018.4.2.3413

Aznar, S., \& Webster, T. (2006). Actividad física y salud en la infancia y la adolescencia. Guía para todas las personas que participan en su educación - Publicaciones - Ministerio de Educación, Cultura y Deporte. (C. de I. y D. educativa Ministerio de Educacion y Cultura, Ed.) (1ra ed.). Madrid, España. https://doi.org/10.4438/351-06-042-1

Brusseau, T. A., Kulinna, P. H., Tudor-Locke, C., Ferry, M., Van Der Mars, H., \& Darst, P. W. (2011). Pedometer-determined segmented physical activity patterns of fourth- and fifthgrade children. Journal of physical activity \& health, 8(2), 279286. https://doi.org/10.1123/jpah.8.2.279

Centers for Disease Control, SHAPE America. Recess. In: Physical activity. U.S. Department of Health \& Human Services. 2017. Recuperado de: https://www.cdc.gov/healthyschools/physicalactivity/recess.htm. Acceso 28 febrero 2020.

De Bourdeaudhuij, I., Verloigne, M., Maes, L., Van Lippevelde, W., Chinapaw, M. J. M., Te Velde, S. J. \& Brug, J. (2013). Associations of physical activity and sedentary time with weight and weight status among 10- to 12-year-old boys and girls in Europe: a cluster analysis within the energy project. Pediatric Obesity, 8(5), 367375. https://doi.org/10.1111/j.2047-6310.2012.00117.x

Delidou, E., Matsouka, O., \& Nikolaidis, C. (2016). Influence of school playground size and equipment on the physical activity of students during recess. European Physical Education Review 22(2): 215-224. https://doi.org/10.1177/1356336x15598790

Esteban, I., Martínez, D., García, L., Ortega, F. B., Delgado, A., Castro, J., \& Veiga O. L. (2017). Objectively Measured Physical Activity During Physical Education and School Recess and Their Associations With Academic Performance in Youth: The UP\&DOWN Study. Journal of Physical Activity and Health, 4: 275282. https://doi.org/10.1123/jpah.2016-0192

Para citar este artículo utilice la siguiente referencia: Bennàsser Torrandell, M.X. y Vidal Conti, J. (2021). Relación entre la actividad física durante el recreo escolar, actividad física semanal y expediente académico. Sportis Sci J, 7 (1), 150-170.

https://doi.org/10.17979/sportis.2021.7.1.6850 http://revistas.udc.es/ 


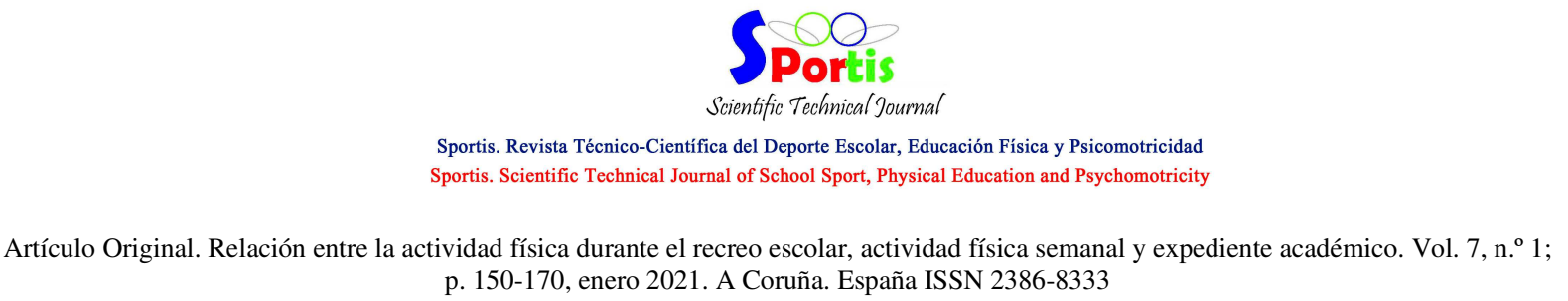
p. 150-170, enero 2021. A Coruña. España ISSN 2386-8333

Govern de les Illes Balears (2018). Estudio sobre la prevalencia de la obesidad en las Illes Balears (EPOIB $\quad$ II). Recuperado de: http://www.caib.es/pidip2front/jsp/adjunto?codi=2175400\&idioma=es

Guthold, R., Stevens, G. A., Riley, L. M., \& Bull, F. C. (2020). Global trends in insufficient physical activity among adolescents: a pooled analysis of 298 population-based surveys with 1.6 million participants. The Lancet. Child \& adolescent health, 4(1), 2335. https://doi.org/10.1016/S2352-4642(19)30323-2

Hall, J., Ochoa, P., Macías, R., Zúñiga, R., \& Sáenz, P. (2018). Actividad física moderada a vigorosa en educación física y recreo en estudiantes de primaria y secundaria de la frontera México-USA. Sportis. Scientific Journal of School Sport, Physical Education and Psychomotricity, 4(3), 426-442. https://doi.org/10.17979/sportis.2018.4.3.3175

Jódar, R. (2003). Revisión de artículos sobre la validez de la prueba de Course navette para determinar de manera indirecta el VO2 max. Revista Internacional de Medicina y Ciencias de la Actividad Física y el Deporte, vol. 3 (11) pp. 173-181. https://doi.org/10.15366/rimcafd

Lamoneda, J. \& Huertas, F. (2017). Análisis de la práctica deportiva-recreativa a través de un programa de promoción en el recreo en función del sexo en adolescentes españoles. Retos, 32: 25-29. https://doi.org/10.5585/podium.v6i3.230

Lizandra, J., Devís, J.,Valencia, A., Tomás, J. M., \& Peiró, C. (2019). Screen time and moderate-to-vigorous physical activity changes and displacement in adolescence: A prospective cohort study. European Journal of Sport Science, 19:5, 686695. https://doi.org/10.1080/17461391.2018.1548649

Léger, L. A., \& Lambert, J. (1982). A maximal multistage 20-m shuttle run test to predict VO2 max. European Journal of Applied Physiology and Occupational Physiology, 49, 112. https://doi.org/10.1007/bf00428958

López, I., Molina, M., Chinchilla, J. L. Garrido, F. J., \& Pascual, C. A. (2017). ¿Son los niños con sobrepeso menos activos durante los recreos escolares? RICYDE. Revista internacional de ciencias del deporte, 47(13), 78-83. https://doi.org/10.5232/ricyde2017.04706

Martínez, J., Aznar, S. \& Contreras, O. (2015). El recreo escolar como oportunidad de espacio y tiempo saludable / The recess school space and time as an opportunity healthy. Revista Internacional de Medicina y Ciencias de la Actividad Física y el Deporte, vol. 15 (59) pp. 419-432. http://cdeporte.rediris.es/revista/revista59/artrecreo596.htm

Martínez, M., Bartolomé, R., Rodríguez, B., Pardo, M. J., \& Martínez, V. (2017). "Football is a boys' game": children's perceptions about barriers for physical activity during recess time.

Para citar este artículo utilice la siguiente referencia: Bennàsser Torrandell, M.X. y Vidal Conti, J. (2021). Relación entre la actividad física durante el recreo escolar, actividad física semanal y expediente académico. Sportis Sci J, 7 (1), 150-170.

https://doi.org/10.17979/sportis.2021.7.1.6850

http://revistas.udc.es/ 


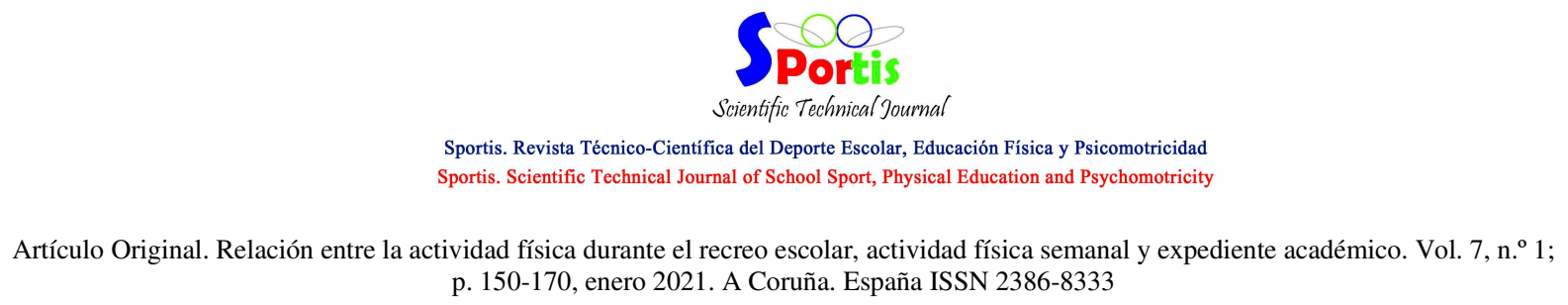
p. 150-170, enero 2021. A Coruña. España ISSN 2386-8333

International Journal of Qualitative Studies on Health and Well-being, 12:1. https://doi.org/10.1080/17482631.2017.1379338

Martínez, V., \& Sánchez, M. (2008). Relación entre actividad física y condición física en niños y adolescentes. Revista Española de Cardiologia, 61, 108111. https://doi.org/10.1157/13116196

Mielgo, J., Aparicio, R., Castillo, A., Ruiz E., Ávila, J. M., Aranceta, J., Gil, A., Ortega, R. M., Serra, LL., Varela, G., \& González, M. (2016) Physical Activity Patterns of the Spanish Population Are Mostly Determined by Sex and Age: Findings in the ANIBES Study. Plosone, 11(2): e0149969. https://doi.org/10.1371/journal.pone.0149969

Ministerio de Sanidad, Consumo y Bienestar Social. (2019). Encuesta Nacional de Salud ENSE, España 2017. Serie informes monográficos \#2 -ACTIVIDAD FÍSICA, DESCANSO Y OCIO. Madrid: Ministerio de Sanidad, Consumo y Bienestar Social.

Ministerio de Sanidad, Servicios Sociales e Igualdad, y Ministerio de Educación, Cultura y Deporte. (2007). Programa PERSEO: estrategia NAOS. Hábitos alimentarios de la población escolar. Cuestionario para los/as niños/as (Exploración física. Análisis de la ingesta alimenticia). Madrid.

Norris, E., Van Steen, T., Direito, A., \& Stamatakis, S. (2019). Physically active lessons in schools and their impact on physical activity, educational, health and cognition outcomes: A systematic review and meta-analysis. Br. J. Sports Med. https://doi.org/10.1136/bjsports2018-100502

Organización Mundial de la Salud. (2017). Estrategia mundial sobre régimen alimentario, $\begin{array}{llll}\text { actividad física } & \text { y } & \text { Ralud. }\end{array}$ de https://www.who.int/dietphysicalactivity/factsheet_inactivity/es

Organización Mundial de la Salud. (2010). Recomendaciones mundiales sobre actividad física para la salud. Ginebra, Suiza.

Ortlieb, S., Schneider, G., Koletzko, S., Berdel, D., Von Berg, A., Bauer, C. P., Herbarth, O., Lehmann, I., Hoffmann, B., Henrich, J., Schulz, H., \& GINIplus and LISAplus Study Groups. (2013). Physical activity and its correlates in children: A cross-sectional study. BMC Public Health, 313-349. https://doi.org/10.1186/1471-2458-13-349

Ortega, F. B., Ruiz, J. R., Hurtig-Wennlöf, A., \& Sjöström, M. (2008). Los adolescentes físicamente activos presentan más probabilidad de una capacidad cardiovascluar saludable independientemente del grado de adiposidad. The European Youth Heart Study. Revista Española de Cardiologia, 61, 123-129. https://doi.org/10.1157/13116199

Para citar este artículo utilice la siguiente referencia: Bennàsser Torrandell, M.X. y Vidal Conti, J. (2021). Relación entre la actividad física durante el recreo escolar, actividad física semanal y expediente académico. Sportis Sci J, 7 (1), 150-170.

https://doi.org/10.17979/sportis.2021.7.1.6850

http://revistas.udc.es/ 


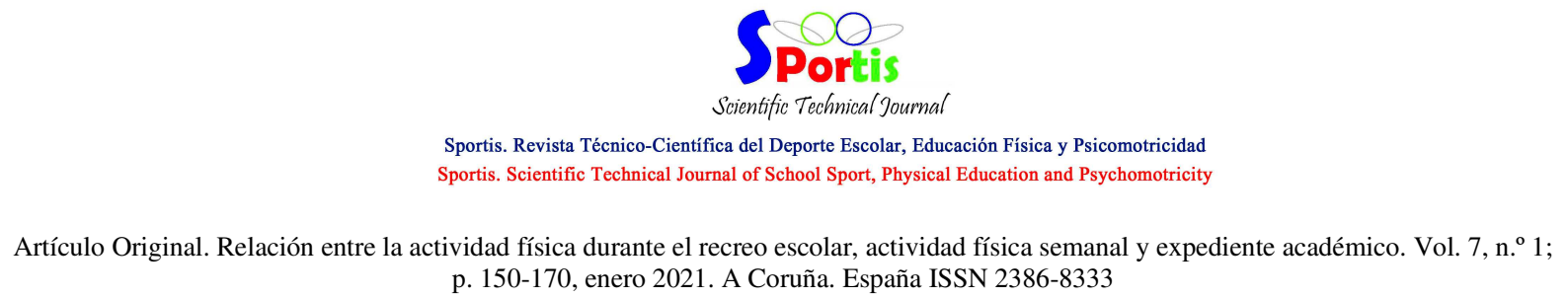
p. 150-170, enero 2021. A Coruña. España ISSN 2386-8333

Parrish, A. M., Okely, A. D., Stanley, R. M., \& Ridgers, N. D. (2013). The effect of school recess interventions on physical activity: a systematic review. Sports Med., 43: 287299. https://doi.org/10.1007/s40279-013-0024-2

Parrish, A., Chong, K. H., Moriarty, A. L. Betterham M., \& Ridgers, N. (2020). Interventions to Change School Recess Activity Levels in Children and Adolescents: A Systematic Review and Meta-Analysis. Sports Med. https://doi.org/10.1007/s40279-020-01347-z

Pawlowski, C. S., Tjørnhøj-Thomsen, T., Schipperijn, J., \& Troelsen, J. (2014). Barriers for recess physical activity: A gender specific qualitative focus group exploration. BMC Public Health, 14(1), 639. https://doi.org/10.1186/1471-2458-14-639

Ridgers, N. D., Stratton, G., \& Fairclough, S. J. (2006). Physical Activity Levels of Children during School Playtime. Sports Med., 36, 359-371. https://doi.org/10.2165/00007256200636040-00005

Salas Sánchez, M., \& Vidal-Conti, J. (2020). Orientaciones para crear patios activos en los centros escolares. Retos, 745753. https://recyt.fecyt.es/index.php/retos/article/view/73038

Savane, F. R., Navarrete, E. M., García de la Hera, M., Giménez, D., González, S., Valera, D., Sempere, M., \& Vioque, J. (2013). Validez del peso y talla auto-referido en población universitaria y factores asociados a las discrepancias entre valores declarados y medidos. Nutrición Hospitalaria, 28, 1633-1638. https://doi.org/10.1016/j.gaceta.2013.12.003

Serrano, J. A., Martí, S., Lera, A., Dorado, C, Gonzalez, J. J., \& Sanchís, J. (2011). Associations between Screen Time and Physical Activity among Spanish Adolescents. PLoS ONE, 6, e24453. https://doi.org/10.1371/journal.pone.0024453

SHAPE America-Society of Health and Physical Educators. Strategies for Recess in Schools. Atlanta, GA: Centers for Disease Control and Prevention, US Dept of Health and Human Services; 2017.

Stanley, R. M., Boshoff, K., \& Dollman, J. (2012). Voices in the playground: A qualitative exploration of the barriers and facilitators of lunchtime play. Journal of Science and Medicine in Sport, 15(1), 44-51. https://doi.org/10.1016/j.jsams.2011.08.002

Viñas, J., \& Pérez, M. (2011). Los hábitos deportivos de la población escolar en España. Madrid: Consejo Superior de Deportes. Recuperado de: http://www.csd.gob.es/csd/estaticos/dep-escolar/encuesta-de-habitos-deportivospoblacionescolar-en-espana.pdf

Para citar este artículo utilice la siguiente referencia: Bennàsser Torrandell, M.X. y Vidal Conti, J. (2021). Relación entre la actividad física durante el recreo escolar, actividad física semanal y expediente académico. Sportis Sci J, 7 (1), 150-170. https://doi.org/10.17979/sportis.2021.7.1.6850 http://revistas.udc.es/ 
Artículo Original. Relación entre la actividad física durante el recreo escolar, actividad física semanal y expediente académico. Vol. 7, n. ${ }^{\circ}$ 1; p. 150-170, enero 2021. A Coruña. España ISSN 2386-8333

Ward, Z. J., Long, M. W., Resch, S. C., Giles, C. M., Cradock, A. L., \& Gortmaker, S. L. (2017). Simulation of growth trajectories of childhood obesity into adulthood. New England Journal of Medicine, 377, 2145-53. https://doi.org/10.1056/nejmoa1703860

Wong, S. L., Leatherdale S. T., \& Manske, S. R. (2006). Reability and validity of a schoolBased Physical Activity Questionnaire. Medicine \& Science in Sports \& Exercise. 38, 1593 1600. https://doi.org/10.1249/01.mss.0000227539.58916.35 